\title{
Thermal property measurements of liquid samples using photoacoustic detection
}

\author{
N. F. Leite and L. C. M. Miranda \\ Laboratório Associado de Sensores e Materiais, Instituto Nacional de Pesquisas Espaciais, Cx. Postal 515. \\ 12201, Săo José dos Campos, SP, Brazil
}

(Received 25 February 1992; accepted for publication 7 April 1992)

In this paper we describe the use of so-called open-photoacoustic cell (OPC) for thermal characterization of transparent liquid samples. The samples used to demonstrate the usefulness of the OPC configuration are water, glycerol, and diffusion pump oil.

\section{INTRODUCTION}

In previous papers ${ }^{1-6}$ we have demonstrated the usefulness of the so-called open photoacoustic cell (OPC) as an alternative detection technique for photoacoustics (PA). The conventional PA detection involves the use of a gas (air) chamber in which a miniature microphone is mounted in one of its walls. The absorbing material is placed inside the air-filled chamber and exposed to modulated light irradiation. As a result of the light-into-heat conversion, following the absorption process within the sample, a pressure fluctuation is produced in the air chamber, which is sensed by the microphone. The proposed OPC technique, on the contrary, requires no additional transducer medium like the air chamber of the conventional PA detection and may be called a minimum-volume PA detection. A similar OPC configuration, still using an additional air chamber but operating in a PA transmission, was described in Ref. 7. The schematic cross section of the OPC configuration is shown in Fig. 1. It consists of mounting the sample directly onto a circular electret microphone. It is an open-cell detection in the sense that the sample is placed on top of the detection system itself, as in the case of piezoelectric and pyroelectric detections. The typical design of an electret microphone $e^{8,9}$ consists of a metallized electret diaphragm (12- $\mu$ m-thick FEP with a 500-1000- $\AA$ deposited metal electrode) and a metal back-plate separated from the diaphragm by an air gap $(45-\mu \mathrm{m}$ long). The metal laycr and the back plate are connected through a resistor $R$. The front sound inlet is a circular hole of 3- $\mathrm{mm}$ diameter, and the front air chamber adjacent to the metallized face of the diaphragm is roughly $1-\mathrm{mm}$ long. As a result of the periodic heating of the sample by the absorption of modulated light, the pressure in the front chamber oscillates at the chopping frequency, causing diaphragm deflections, which generate a voltage $V$ across the resistor $R$. This voltage is subsequently fed into a field-effect transistor (FET) preamplifier already built in the microphone capsule.

This minimal-volume PA detection has been applied in the last two years to the characterization of both thermal and transport properties of several samples. These applications include the monitoring of adhesive curing, ${ }^{3}$ the measurement of thermal diffusivity of doped polymers, ${ }^{4,5}$ twolayer systems, ${ }^{10}$ PbSnTe semiconductors, ${ }^{6}$ as well as to electronic transport properties of $\mathrm{Si}$ and $\mathrm{GaAs} .{ }^{11,12}$ In this paper, we explore the enhanced signal-to-noise ratio of the OPC detection to the complete thermal characterization of transparent liquid samples by measuring their thermal effusivities and diffusivities. The thermal effusivity $c$ and diffusivity $\alpha$ are defined by $e=\sqrt{k \rho c}$ and $\alpha=k / \rho c$, where $k$ is the thermal conductivity, $\rho$ the density, and $c$ is the specific heat. As can be seen from the above definitions, the knowledge of $e=k / \sqrt{\alpha}$ and $\alpha$ completely determine the thermal conductivity and heat capacity of a given material.

\section{THEORY}

Consider the schematic cross section for the electret microphone shown in Fig. 2, in which the electret foil has a charge density $\chi$ per $\mathrm{cm}^{2}$, dielectric constant $\epsilon$, thickness $l_{m}$, and is separated from the metal back plate by an air gap of thickness $l_{b}$. Denoting by $\delta P \exp (j \omega t)$ the PA pressure fluctuation generated in the front air chamber, due to the periodic heating of the samplc, it follows from the theory of electret microphones ${ }^{8,13}$ that the microphone output voltage $V$, for modulation frequencies well below the resonance frequency, can be written as

$$
V=\frac{l_{b} l_{m} \chi}{l_{b} \epsilon+l_{m} \epsilon_{0}} \frac{j \omega R C}{1+j \omega R C} \frac{\delta P}{\gamma P_{0}} e^{j \omega t},
$$

where $C=A \epsilon \epsilon_{0} /\left(l_{m} \epsilon_{0}+l_{b} \epsilon\right)$ is the capacitance of the microphone of area $A, P_{0}$ is the ambient pressure, $\gamma$ is the air specific heat ratio, and $\omega=2 \pi f$ with $f$ being the modulation frequency.

To find the pressure fluctuation $\delta P$ in the front air chamber of the OPC cell we assume the RosencwaigGersho $^{14}$ model for the production of the PA signal. According to this model, the pressure fluctuation in the PA effect is solely due to the heat flow (resulting from the light absorption) into the PA chamber. Assuming that the air in the PA chamber responds adiabatically, the acoustic pressure in the OPC configuration is given by

$$
\delta P=\frac{\gamma P_{0}}{T_{0}}\left\langle T_{y}\right\rangle
$$

where $T_{0}$ is the ambient temperature, and $\left\langle T_{g}\right\rangle$ is the spatially averaged temperature fluctuation of the air in the PA chamber. This temperature fluctuation is, in turn, obtained by solving the thermal diffusion equation for the sample-air chamber system. 


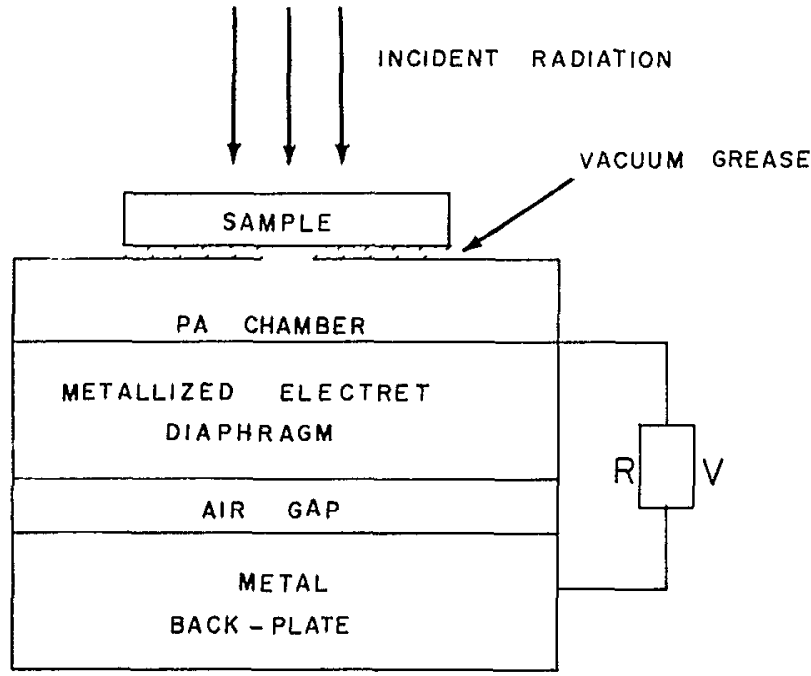

FIG. 1. Cross section of the open-photoacoustic cell using the front air chamber of a common electret microphone as a transducer medium.

Consider the transparent liquid sample holder shown schematically in Fig. 3(a). It consists of an acrylic ring of thickness $l_{s}$ whose bottom is closed by a $12-\mu$ m-thick $\left(l_{0}\right)$ Al foil disk. The liquid sample is placed inside the ring and the entire sample holder is placed on top of the microphone. For the case of a transparent sample, illuminated by nonabsorbing modulated light, the periodic heat is deposited at the surface of the Al foil in contact with the liquid sample as indicated in Fig. 3(b) at $x=-l_{0}$. The thermal diffusion equations for the OPC configuration of Fig. 3(b) are

$$
\begin{aligned}
& \frac{\partial^{2} T_{s}}{\partial x^{2}}=\sigma_{s}^{2} T_{s} \\
& \frac{\partial^{2} T_{0}}{\partial x^{2}}=\sigma_{0}^{2} T_{0}-\frac{\beta^{\prime} I_{0}}{k_{0}} \delta\left(x+l_{0}\right), \\
& \frac{\partial^{2} T_{g}}{\partial x^{2}}=\sigma_{g}^{2} T_{g}
\end{aligned}
$$

where $\sigma_{i}=(1+j) a_{i}$ with $a_{i}=\left(\omega / 2 \alpha_{i}\right)^{1 / 2}$ and $j=\sqrt{-1}$. Here, the index $i$ denotes the sample $(i=s)$, the Al foil $(i=0)$, and the air in the PA chamber $(i=g)$. In Eqs.

\section{METAL LAYER ELECTRET}

AIR GAP

METAL BACK PLATE

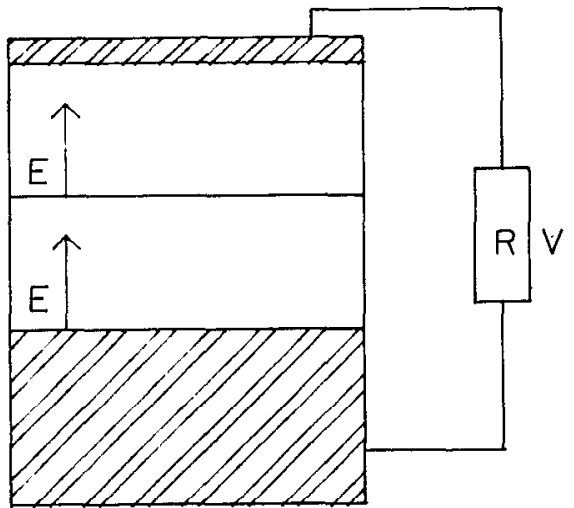

FIG. 2. Cross section for the electret microphone.

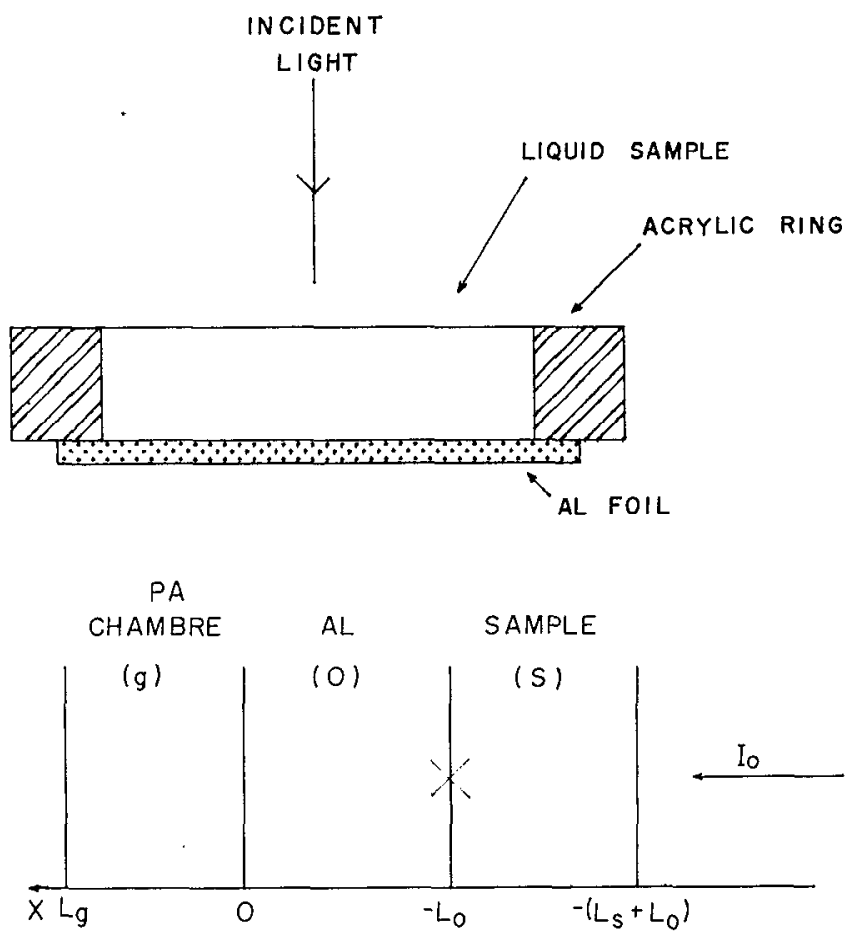

FIG. 3. (a) Sample holder for transparent liquid. (b) Schematic openphotoacoustic cell geometry for transparent liquid.

(3)-(5), we have assumed that the light beam with intensity $I_{0}$ is absorbed at $x=-l_{0}$, and $\beta^{\prime}$ denotes the surface absorption coefficient. Solving Eqs. (3)-(5) together with the boundary conditions of temperature and heat-flux continuity, and calculating the average temperature, $\left(T_{g}\right)$, in the PA chamber between $x=0$ and $x=l_{g}$, one finds, for a thermally thick sample,

$$
\delta P=\frac{\gamma P_{0} \beta^{\prime} I_{0}}{T_{0} l_{g} \sigma_{g} k_{0} \sigma_{0}} \frac{e^{j \omega t}}{b \cosh \left(l_{0} \sigma_{0}\right)+\sinh \left(l_{0} \sigma_{0}\right)},
$$

where $b=k_{s} \sigma_{s} / k_{0} \sigma_{0}$. Noting that an $\mathrm{Al}$ foil with a thickness of roughly $12 \mu \mathrm{m}$ is thermally thin (i.e., $l_{0} \sigma_{0}<1$ ) for modulation frequencies $f$ up to a few $\mathrm{MHz}$, one can rewrite (6) as

$$
\delta P=\frac{\gamma P_{0} \beta^{\prime} I_{0} \alpha_{g}^{1 / 2} \alpha_{s}^{1 / 2}}{2 \pi T_{0} l_{g} k_{s} f} e^{j(\omega t-\pi / 2)} .
$$

Equation (7) tells us that the PA signal of a transparent liquid sample varies as $f^{-1}$ and is proportional to ratio $\sqrt{\alpha_{s}} / k_{s}=e_{s}^{-1}$.

In contrast, when the sample holder is empty, it can be shown that the pressure fluctuation $\delta p$ is given by

$$
\delta P_{0}=\frac{\gamma P_{0} \beta^{\prime} I_{0} \alpha_{g}^{1 / 2} \alpha_{0}}{(2 \pi)^{3 / 2} T_{0} l_{g} l_{0} k_{0} f^{3 / 2}} e^{j(\omega t-3 \pi / 4)}
$$

We call this the reference signal. Equation (8) tells us that the reference signal varies as $f^{-3 / 2}$ and is proportional to the ratio $\alpha_{0} / k_{0}$.

It follows from Eqs. (7) and (8) that in order to find the sample's thermal effusivity all we have to do is to measure the sample signal, as given by Eq. (7), as well as the 


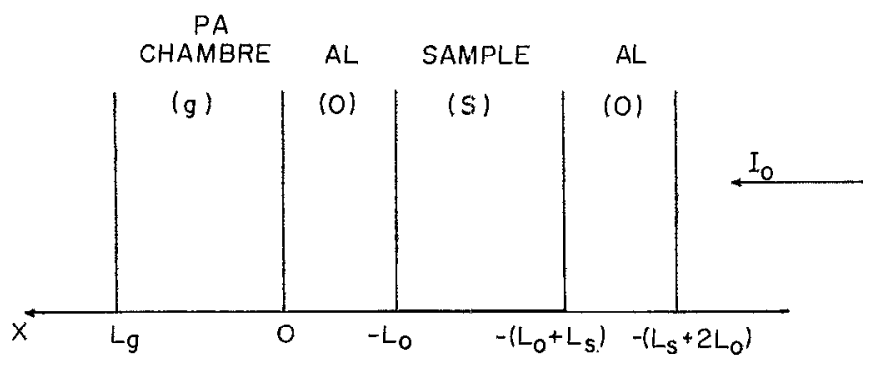

FIG. 4. Schematic open-photoacoustic cell geometry for transparent liquid sample thermal diffusivity measurements.

empty cell signal, and calculate the ratio $S_{R}$ of the two signal amplitudes, namely,

$$
S_{R}=\frac{l_{0} \rho_{0} c_{0}}{e_{s}} \sqrt{\omega}
$$

In other words, since $l_{0} \rho_{0} c_{0}$ is well known for the Al foil, knowing the slope of the signal amplitude ratio $S_{R}$, as a function of the square root of the modulation frequency, one can determine $e_{s}$.

The thermal diffusivity can be measured using the standard OPC procedure. ${ }^{2-5}$ It consists of using essentially the same type of sample holder with the additional use of another thin $\mathrm{Al}$ foil covering the liquid sample, as indicated in Fig. 4. The use of this Al cover foil serves as a localized heat source at the outer surface [at $x=-\left(l_{s}+l_{0}\right)$ ] of the liquid sample. The heat generated at the $12-\mu \mathrm{m} \mathrm{Al}$ cover foil takes a few microseconds to cross, leading essentially to an instantaneous heat source at $x=-\left(l_{s}+l_{0}\right)$ for the liquid sample. Performing similar calculations to the ones indicated above for this new configuration, one finds

$$
\begin{aligned}
\delta P= & \frac{\gamma P_{\alpha} \beta^{\prime} I_{0}}{T_{0} l_{g} \sigma_{g} k_{0} \sigma_{0}} \\
& \times \frac{e^{j \omega t}}{b \cosh \left(l_{0} \sigma_{0}\right) \sinh \left(l_{s} \sigma_{s}\right)+\sinh \left(l_{0} \sigma_{0}\right) \cosh \left(l_{s} \sigma_{s}\right)},
\end{aligned}
$$

which, for the case of a thermally thin Al foil, $l_{0} \sigma_{0}<1$ (which for a $12-\mu \mathrm{m} \mathrm{Al}$ foil is valid for modulation frequencies up to the $\mathrm{MHz}$ region) reduces to

$$
\delta P=\frac{\gamma P_{0} \beta^{\prime} I_{0}}{T_{0} l_{g} \sigma_{g} k_{s} \sigma_{s}} \frac{e^{j \omega t}}{\sinh \left(l_{s} \sigma_{s}\right)} .
$$

For high modulation frequencies such that the sample is thermally thick, $l_{s} \sigma_{s}>1$, Eq. (11) reduces to

$$
\delta P=\frac{\gamma P_{0} \beta^{\prime} I_{0} \alpha_{g}^{1 / 2} \alpha_{s}^{1 / 2}}{\pi T_{0} l_{g} k_{s} f} e^{-a \sqrt{f}} e^{i(\omega t-\pi / 2-a \sqrt{f})},
$$

where $a=l_{s}\left(\pi / \alpha_{s}\right)^{1 / 2}$. Equation (12) means that the signal decays exponentially with increasing modulation frequency. The thermal diffusivity is calculated from the coefficient $a$ of the exponential decay of the signal amplitude as a function of $\sqrt{f}$.

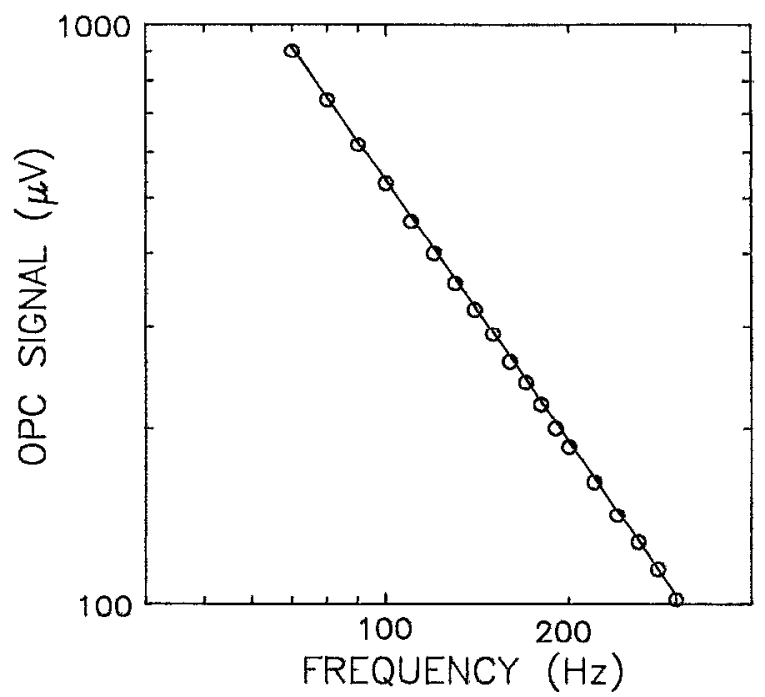

FIG. 5. OPC signal amplitude as a function of the modulation frequency for the empty sample holder. The solid line represents the best fit of the data to the expression $A / f^{-1.5}$.

\section{EXPERIMENT}

The thermal effusivity measurements where performed using a 0.45 -mm-thick acrylic ring of 3-mm i.d., as sample holder. The modulated light source was provided by a 250 W tungsten filament lamp whose beam after being heatfiltered was focused at the bottom $12-\mu \mathrm{m} \mathrm{Al}$ foil closing the sample holder, as indicated in Fig. 3(a). A lock-in amplifier was used to analyze the amplitude and phase of the microphone output signal. The samples used were distilled water, glycerol, and diffusion pump (DC 704) oil. In Figs. 5 and 6 we show the OPC signal amplitudes for the empty sample holder and for water, as a function of the modulation frequency, respectively. As predicted by Eq. (8) the empty sample holder varies as $f^{-1.5}$; the solid line of Fig. 5 represents the signal amplitude data fitting to the expres-

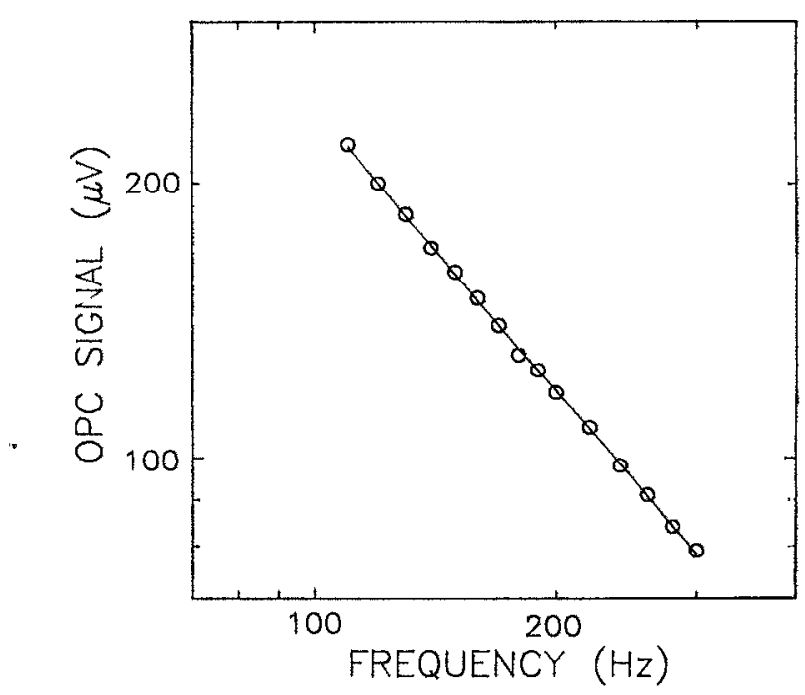

FIG. 6. OPC signal amplitude as a function of the modulation frequency for water. The solid line represents the best fit of the data to the expression $B / f$. 


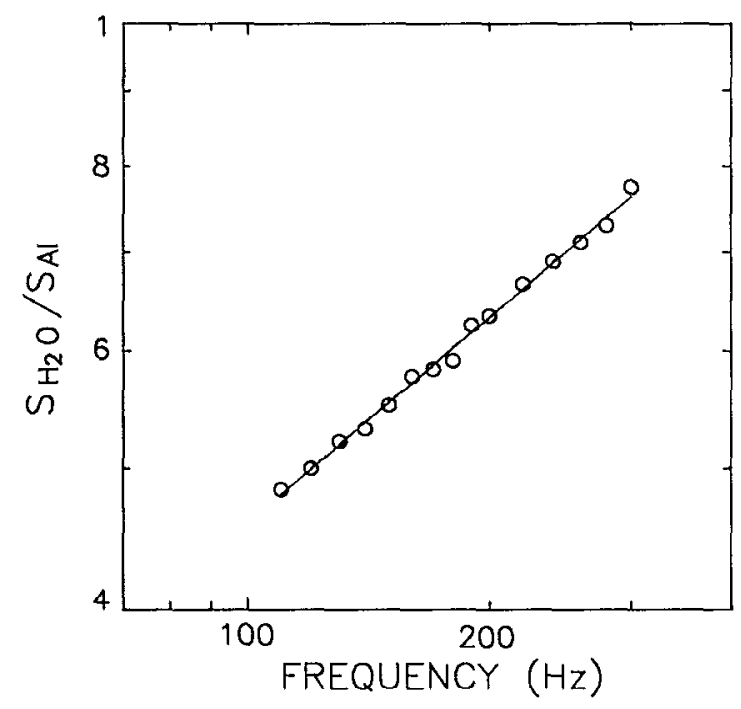

FIG. 7. Ratio of the water to the Al foil signal amplitude. The solid line represents the best fit of the data to $\mathrm{Eq}$. (7).

sion $A / f^{-1.5}$. In contrast the signal amplitude data for water behaves as $f^{-1.0}$ in agreement with Eq. (7); the solid curve in Fig. 6 represents the water signal amplitude data fitting to the expression $B / f$.

In Fig. 7 we show the ratio of the water to the $\mathrm{Al}$ foil signal amplitude data as a function of the modulation frequency. As predicted by Eq. (9) this signal amplitude ratio should vary as $\sqrt{f}$. The solid line in this figure is the result of the ratio data fitting to the expression $C \sqrt{f}$, where, according to Eq. (9), the fitting parameter $C$ is equal to $l_{0} \rho_{0} c_{0} / e_{5}$. From the data fitting result and using the known values of $l_{0} \rho_{0} c_{0}$ for the Al foil, namely $l_{0}=12 \mu \mathrm{m}, \rho_{0} c_{0}$ $=2.429 \times 10^{6} \mathrm{~J} / \mathrm{m}^{3} \mathrm{~K}$ we found the water thermal effusivity, $e_{s}=(1.61 \pm 0.11) \times 10^{3} \mathrm{~J} / \mathrm{m}^{2} \mathrm{~s}^{1 / 2} \mathrm{~K}$. This value of $e_{s}$ compares quite well with the literature value, namely, 1563 $\mathrm{J} / \mathrm{m}^{2} \mathrm{~s}^{1 / 2} \mathrm{~K}$. The same procedure was also carried out with the other testing samples and the results are summarized in Table I. It follows from Table I that the values obtained for the thermal effusivity using the OPC technique agree quite well with the ones quoted in the literature.

Next, instead of using the straightforward method for measuring the thermal diffusivity, as outlined above, we have measured directly the specific heat using the temperature rise technique under continuous light illumination. The liquid was placed inside of a 2.5 -mm-thick acrylic ring of $10-\mathrm{mm}$ i.d. with $60-\mu \mathrm{m}$-thick $\mathrm{Al}$ foils closing both sides of the ring. The $\mathrm{Al}$ foils were sprayed with black paint (with an emissivity of 0.99 ) to ensure not only a good light absorbing surface but also the same heat transfer coefficient for each surface. The samples were adiabatically suspended

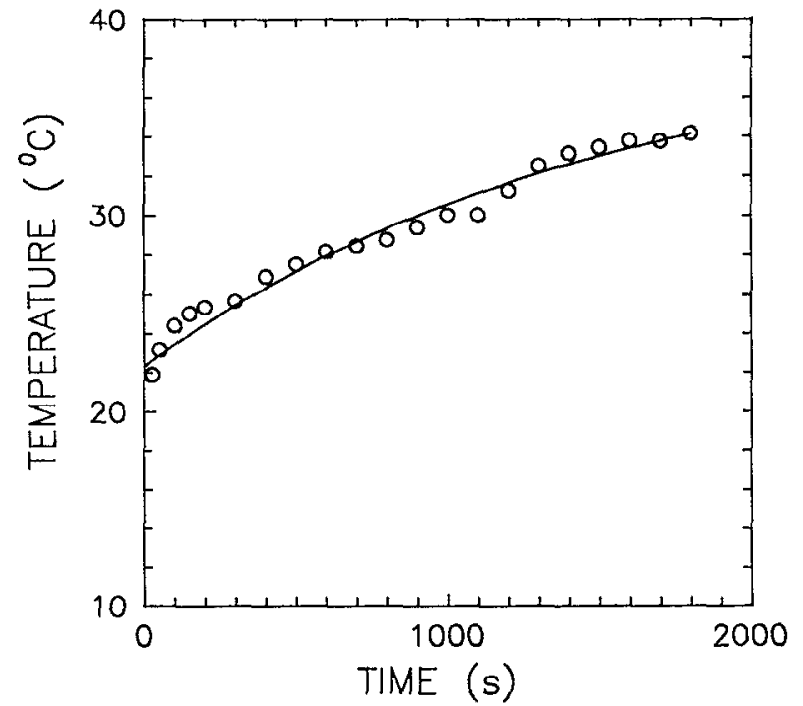

FIG. 8. Back-surface temperature evolution of a 2 -mm-thick ring with water as a function of time under continuous white light illumination. The solid line represents the best fit of the data to Eq. (11) of the text.

in a Dewar which was subsequently vacuum sealed. Under these conditions the main heat-loss mechanism is the loss by radiation. The Dewar had an entrance optical glass window through which the continuous white-light beam was focused onto one of the sample surfaces. On the opposite surface we had attached a thermocouple to the sample using thermal paste; in this way, the temperature evolution of the back surface could be monitored as a function of time. Care has been taken to prevent the heating light from reaching the thermocouple. Since the sample thickness, typically of the order of $3 \mathrm{~mm}$, were much smaller than their widths (e.g., $1 \mathrm{~cm}$ ), the simple one-dimensional heat diffusion equation with radiation losses could be applied to our experiment.

Solving the one-dimensional heat diffusion equation ${ }^{15}$ it can be shown that the long-term time evolution (i.e., four limes larger than the heat diffusion time $\sim l^{2} / \alpha$ ) of the back-surface temperature rise is given by

$$
\Delta T=\frac{I_{0} \alpha \tau}{l k}\left(1-e^{-t / \tau}\right),
$$

where $I_{0}$ is the intensity of the incident light beam, and $\tau=l k / 2 \alpha H$ is the rising time. Here, $H=4 \sigma T_{0}^{3}$, where $\sigma$ is the Stefan-Boltzmann constant and $T_{0}$ is the ambient temperature, is the radiation and heat-transfer coefficient. In Fig. 8, the back-surface temperature rise of water as a function of time. The solid line in Fig. 8 represents the result of the best fit of the experimental data to Eq. (13).

TABLE I. Thermal effusivities obtained using the OPC detection and literature values, specific heat and thermal conductivities obtained using the proposed technique.

\begin{tabular}{lcccc}
\hline \hline Sample & $e_{s}\left(\mathrm{~J} / \mathrm{m}^{2} \mathrm{~s}^{1 / 2} \mathrm{~K}\right)$ & $e\left(\mathrm{~J} / \mathrm{m}^{2} \mathrm{~s}^{1 / 2} \mathrm{~K}\right)$ & $\rho c\left(\mathrm{~J} / \mathrm{m}^{3} \mathrm{~K}\right)$ & $k(\mathrm{~W} / \mathrm{mK})$ \\
\hline Water & $(1.61 \pm 0.11) \times 10^{3}$ & 1563 (Ref. 16) & $(4.28 \pm 0.10) \times 10^{6}$ & 0.610 \\
Glycerol & $(1.00 \pm 0.11) \times 10^{3}$ & 932 (Ref. 7) & $(2.913 \pm 0.046) \times 10^{6}$ & 0.347 \\
Dif. pump oil & $(0.59 \pm 0.12) \times 10^{3}$ & 447 (Ref. 18) & $(2.165 \pm 0.017) \times 10^{6}$ & 0.163 \\
\hline
\end{tabular}


From the adjusted value of $\tau$ we have calculated the water specific heat: the value obtained within a $2.5 \%$ error in the data fitting was $4.280 \times 10^{6} \mathrm{~J} / \mathrm{m}^{3} \mathrm{~K}$. The same procedure for measuring the specific heat was adopted for the other samples. The results are shown in Table I. Using these values together with the ones for the thermal effusivities shown in Table I we have obtained the thermal conductivities of our samples. The results are also summarized in Table I. In particular, we note that for the diffusion pump oil DPO, the only parameter available to us was its density. The values we have found for both $k$ and $c$ are, however, in good agreement ${ }^{18}$ with those of similar samples, such as, apiezon and silicone oils.

The good agreement between the measured and literature values obtained for thermal conductivities and specific heat shows that the proposed minimum-volume method is a simple and accurate technique for the complete thermal characterization of transparent liquids. Its advantages over the conventional PA techniques are the use of a minimal gas chamber and, consequently, an increased signal-to-noise ratio, and its adaptability to practical restriction imposed by experimental system requirements, especially when minimum preparation is required. The accuracy of the thermal effusivity measurements can be further improved if the small changes in the light reflections from the empty and the liquid-filled cell measurements are accounted for. This is most effectively done by using a laser light source and correcting the effective absorption coefficient with the calculated reflectivities.
${ }^{1}$ M. D. Silva, I. N. Bandeira, and L. C. M. Miranda, J. Phys. E 20, 1476 (1979).

${ }^{2}$ L. F. Perondi and L. C. M. Miranda, J. Appl. Phys. 62, 2955 (1987).

${ }^{3}$ A. Torres-Filho, L. F. Perondi, and L. C. M. Miranda, J. Appl. Polym. Sci. 35, 103 (1988).

${ }^{4}$ N. F. Leite, A. Torres-Filho, R. Stempniak, and L. C. M. Miranda, J. Appl. Phys. 66, 407 (1989).

${ }^{5}$ A. Torres-Filho, N. F. Leite, L. C. M. Miranda, N. Cella, and H. Vargas, J. Appl. Phys. 66, 97 (1989).

${ }^{6}$ S. O. Ferreira, C. Ying An, I. N. Bandeira, and L. C. M. Miranda, Phys. Rev. B 39, 7969 (1989).

${ }^{7}$ S. O. Kanstad and P. E. Nordal, Opt. Commun. 26, 367 (1978).

${ }^{8}$ G. M. Sessler, J. Acoust. Soc. Am. 35, 1354 (1963).

${ }^{9}$ G. M. Sessler and J. E. West, in Electrets, edited by G. M. Sessler (Springer, Berlin, 1980), Spring Series in Topics in Applied Physics, Vol. 33 , p. 347.

${ }^{10}$ A. Mansanares, A. C. Bento, H. Vargas, N. F. Leite, and L. C. M. Miranda, Phys. Rev. B 42, 4477 (1990).

${ }^{11}$ A. Pinto-Neto, H. Vargas, N. F. Leite, and L. C. M. Miranda, Phys. Rev. B 40, 3294 (1989).

${ }^{12}$ A. Pinto-Neto, H. Vargas, N. F. Leite, and L. C. M. Miranda, Phys. Rev. B 41, 9971 (1990).

${ }^{13}$ P. M. Morse, in Vibration and Sound, in International Series in Pure and Applied Physics, edited by L. I. Schiff (MacGraw-Hill, New York, 1948).

${ }^{14}$ A. Rosencwaig and A. Gersho, J. Appl. Phys. 47, 2383 (1976).

${ }^{15}$ H. S. Carslaw and J. C. Jaeger, Conduction of Heat in Solids (Clarendon, Oxford, 1973).

${ }^{16}$ R. C. Weast, ed., Handbook of Chemistry and Physics (The Chemical Rubber Co., Cleveland, 1971-1972).

${ }^{17}$ Y. S. Touloukian, R. W. Powell, C. Y. Ho, and M. C. Nicolasu, in Thermal Diffusivity (IFI/Plenum, New York, 1973).

${ }^{18}$ L. Holland, W. Steckelmacher, and J. Yarwood, eds., Vacuum Monual (E. \& F. N. Spon, London, 1974) 